FEDERAL

RESERVE

BANK of

ST. LOUIS
RESEARCH DIVISION

Working Paper Series

\title{
Forecasting National Recessions Using State Level Data
}

\author{
Michael T. Owyang, \\ Jeremy M. Piger \\ and \\ Howard J. Wall
}

\author{
Working Paper 2012-013B \\ https://doi.org/10.20955/wp.2012.013
}

November 2013

\author{
FEDERAL RESERVE BANK OF ST. LOUIS \\ Research Division \\ P.O. Box 442 \\ St. Louis, MO 63166
}

The views expressed are those of the individual authors and do not necessarily reflect official positions of the Federal Reserve Bank of St. Louis, the Federal Reserve System, or the Board of Governors.

Federal Reserve Bank of St. Louis Working Papers are preliminary materials circulated to stimulate discussion and critical comment. References in publications to Federal Reserve Bank of St. Louis Working Papers (other than an acknowledgment that the writer has had access to unpublished material) should be cleared with the author or authors. 


\title{
Forecasting National Recessions Using State Level Data*
}

\author{
Michael T. Owyang ${ }^{\dagger} \quad$ Jeremy Piger ${ }^{\ddagger}$ Howard J. Wall ${ }^{\S}$
}

This draft: November 8, 2013

\begin{abstract}
A large literature studies the information contained in national-level economic indicators, such as financial and aggregate economic activity variables, for forecasting and nowcasting U.S. business cycle phases (expansions and recessions.) In this paper, we investigate whether there is additional information useful for identifying business cycle phases contained in subnational measures of economic activity. Using a probit model to forecast the NBER expansion and recession classification, we assess the incremental information content of state-level employment growth over a commonly used set of national-level predictors. As state-level data adds a large number of predictors to the model, we employ a Bayesian model averaging procedure to construct forecasts. Based on a variety of forecast evaluation metrics, we find that including state-level employment growth substantially improves nowcasts and very short-horizon forecasts of the business cycle phase. The gains in forecast accuracy are concentrated during months of national recession.
\end{abstract}

Keywords: turning points, probit, covariate selection

JEL Classification Numbers: C52, C53, E32, E37

${ }^{*}$ We thank the editor, two anonymous referees, and seminar participants at the University of Houston, Federal Reserve Bank of St. Louis, and University of Texas, El Paso for helpful comments. This paper also benefited from conversations with Graham Elliott and Oscar Jordá. Kristie M. Engemann and Kate Vermann provided research assistance. The views expressed herein do not reflect the official positions of the Federal Reserve Bank of St. Louis or the Federal Reserve System.

${ }^{\dagger}$ Research Division, Federal Reserve Bank of St. Louis, (Michael.T.Owyang@stls.frb.org)

${ }^{\ddagger}$ Department of Economics, University of Oregon, (jpiger@uoregon.edu)

$\S$ Department of Economics, Lindenwood University, (hwall@lindenwood.edu) 


\section{Introduction}

A traditional view of the U.S. business cycle is that of alternating phases of expansion and recession, where expansions correspond to widespread, persistent growth in economic activity, and recessions consist of widespread, relatively rapid, decline in economic activity. ${ }^{1}$ A large literature investigates different aspects of these business cycle phases and documents asymmetries across them. Such work experienced a resurgence following Hamilton (1989), who built a modern statistical model of the alternating phases characterization of the business cycle by describing the latent business cycle phase as following a first-order Markov process that influences the mean growth rate of output.

Timely identification of business cycle phases, and the associated turning points between them, is of particular interest to academics, policymakers, and practitioners. A substantial literature has investigated the extent to which the business cycle phase can be predicted using a variety of economic and financial time series. ${ }^{2}$ Here, the forecasting problem is to use data available at time period $t$ to predict whether period $t+h$ will be an expansion or recession period. The most ambitious task is of course to predict the future business cycle phase at long horizons, and the literature has had some limited success predicting recessions one-year ahead using a predictor that measures the slope of the yield curve. For forecasting the business cycle phase at short horizons, predictors measuring aggregate real activity, such as employment or output growth, are found to be valuable. Not surprisingly, this is especially true for $h=0$ predictions, which are typically referred to as "nowcasts." 3

Although they have received less attention in the literature, short-horizon forecasts and nowcasts of business cycle turning points are of considerable interest. While long horizon forecasts of the business cycle phase would give economic agents the most advance warning

\footnotetext{
${ }^{1}$ See Mitchell (1927) and Burns and Mitchell (1946).

${ }^{2}$ See e.g., Estrella (1997), Estrella and Mishkin (1998), Kauppi and Saikkonen (2008), Rudebusch and Williams (1991) and Berge (2013). For a recent summary of this literature, see Katayama (2008).

${ }^{3}$ Here, the prefix "now" in nowcast refers to the use of data available in period $t$ to evaluate whether period $t$ is a recession or expansion period. Note that because of data reporting lags, some of the data available in period $t$ will be measuring a period prior to month $t$.
} 
of a new business cycle turning point, it has historically been the case that new business cycle phases have not been forecasted accurately. Indeed, in most cases new phases have only been identified in real time after many months have passed following the turning point. ${ }^{4}$ As a recent example of this, Hamilton (2011) surveys a wide range of statistical models that were in place to nowcast business cycle turning points using aggregate data, and finds that such models did not send a definitive signal regarding the December 2007 NBER peak until late 2008. Improved short-horizon forecasts and nowcasts thus hold the promise of giving a quicker signal that a new recession or expansion is imminent or has already begun. These could improve the speed of both policy and private sector adjustments to this new business cycle phase.

The existing literature has focused on the use of predictors measured at the national level. However, there is reason to believe that variables measured at the subnational level would be useful for identifying the national business cycle phase. Owyang et al. (2005) and Hamilton and Owyang (2011) provide evidence that the business cycles of individual U.S. states are often out of phase with that of the nation. If these phase shifts are systematic, then incorporating data from leading regions may be useful for predicting the national business cycle phase. Also, even if subnational regions are coincident with the aggregate business cycle phase, there may be regions that experience recession and expansion phases more severely than the nation as a whole. In these cases, incorporating data from these regions will provide a stronger signal regarding the business cycle phase that is currently in operation, and will thus improve nowcasts and short-horizon forecasts of the business cycle phase. ${ }^{5}$

In this paper, we assess whether state-level economic indicators contain incremental information useful for forecasting and nowcasting U.S. business cycle phases. Following the bulk

\footnotetext{
${ }^{4}$ Since the inception of the NBER's business cycle dating committee in 1978, the NBER has announced new business cycle peaks with a lag of 7.5 months and troughs with a lag of 15 months. Chauvet and Piger (2008) show that statistical models estimated using aggregate data improve on the NBERs timeliness for troughs, but not for peaks.

${ }^{5}$ Short-horizon forecasts will be improved because of the persistence of business cycle phases. If subnational data sends a quicker signal that a new business cycle phase has begun, then we will have more accurate short-horizon forecasts of the future business cycle phase.
} 
of the literature, we take the NBER's chronology of the dates of U.S. business cycle phases as given. We then construct a monthly probit model to attach probabilities of expansion and recession to current and future periods, which incorporates both national- and state-level variables as predictors. The national variables we use are those found to be good predictors of national business cycle phases at various horizons by the existing literature. In particular, we focus on interest rates, asset prices, aggregate employment, and aggregate industrial production. To these, we add state-level employment growth to capture the predictive ability of subnational economic activity measures.

By using state-level employment growth, we measure subnational economic activity with a coincident indicator, rather than a leading indicator. This raises the question of what hope such data would have for helping predict the aggregate business cycle phase? While state employment growth is a coincident indicator, it is likely an indicator that is coincident with that state's business cycle phase. Thus, as discussed above, if a state's business cycle leads the national business cycle phase, then state employment growth could be a leading indicator for the national business cycle phase. As evidence of this, Hernandez-Murillo and Owyang (2006) show that adding regional employment data can assist in predicting aggregate employment growth in the United States. Also, even if state employment growth is coincident with the national level business cycle phase, state employment growth may provide improved nowcasts and short-horizon forecasts of the aggregate business cycle phase if the employment response to the business cycle phase in operation is relatively strong for certain states. ${ }^{6}$

Despite the potential promise of state-level data for improving nowcasts and forecasts of

\footnotetext{
${ }^{6}$ Our choice to focus on state-level employment growth is also driven by the lack of suitable leading indicators measured at the state level. The state-level leading index produced by the Federal Reserve Bank of Philadelphia is only available in real-time for less than ten years. Because this series was constructed in-sample specifically to maximize its ability to predict state-level economic activity, using it in a pseudo out-of-sample forecasting exercise could yield very distorted results. See Diebold and Rudebusch (1991) for a similar point made for the national index of leading indicators. Also, only two of the four components of the index are measured at the state level, and neither of these is available until after the early 1980s recession. Given the relative scarcity of recessions over this period, this would not allow us to both estimate the model and perform an out-of-sample forecasting experiment over a reasonable number of recessions.
} 
business cycle phases, simply adding this data into the information set used in a forecasting model is problematic. It is likely that many states will not be informative about future national business cycle phases at all, or perhaps any, forecast horizons. Further, there is significant collinearity in employment growth across U.S. states. Put together, the naive use of all state-level data will likely lead to an overparameterized model with a high level of estimation uncertainty, which will not bode well for improved forecasting performance. One may reduce, though not eliminate, these problems by aggregating across states to the regional level. However, this aggregation would potentially average states that contain very different forecasting information.

In this paper, we take a Bayesian model averaging (BMA) approach to incorporate statelevel predictors in a forecasting model. In particular, we explicitly incorporate the selection of predictors into the estimation of the model, and average forecasts across models with different sets of predictors by constructing the posterior predictive distribution for the future business cycle phase. This approach allows individual states with predictive content for the business cycle phase at a particular horizon to be highlighted in producing forecasts, while pushing out those states that are not informative. Notably, the Bayesian approach to constructing forecasts also incorporates uncertainty regarding model parameters.

Based on a variety of forecast evaluation metrics, we find that including state-level employment growth significantly improves nowcasts and very short-horizon forecasts of the NBER business cycle phase over those produced by a model using only national-level data. We document the incremental information content of the state-level data based on the model's out-of-sample forecast performance over the past 30 years. We also show that the forecasting improvement comes primarily from improved classification of recession months. To give two examples, for one-month ahead forecasts, $23 \%$ of recession months are correctly classified using the model that includes state-level data, but not by the model based on national-level data only. Also, again based on one-month ahead forecasts, the December 2007 business cycle peak would have been identified by late February 2008 when using both 
state and national-level data, as opposed to late July 2008 when using only national-level data.

The balance of the paper is as follows: Section 2 outlines the empirical model used for forecasting recessions and describes the Bayesian approach to estimation and construction of forecasts. Section 3 describes the national- and state-level data used to estimate the model and evaluate forecasts. Section 4 presents the results for both in-sample model fit and the performance of out-of-sample forecasts. Section 5 summarizes and concludes.

\section{Empirical Approach}

\section{$2.1 \quad$ Model}

Define $S_{t} \in\{0,1\}$ as a binary random variable that indicates whether month $t$ belongs to an expansion (0) or recession (1) phase. Our objective is to forecast the business cycle phase, $S_{t+h}$, based on information available to a forecaster at the end of month $t$. This information may include national-, state-, or regional-level variables and is collected in the $n \times 1$ vector $X_{t}$.

Following the bulk of the existing literature, we use a probit model to link $S_{t+h}$ to $X_{t}$. Here, the probability that $S_{t+h}=1$ is given by:

$$
\operatorname{Pr}\left[S_{t+h}=1 \mid \rho\right]=\Phi\left(\alpha+X_{t}^{\prime} \beta\right)
$$

where the link function, $\Phi($.$) , is the standard normal cumulative density function, \beta$ is an $n \times 1$ vector of coefficients, and $\rho=\left[\alpha, \beta^{\prime}\right]^{\prime}$ is an $(n+1) \times 1$ vector holding the model parameters.

The number of potentially relevant forecasting variables available in $X_{t}$ may be large. This is especially true with the inclusion of subnational data, as variables are measured repeatedly across regions or states. From a forecasting perspective, this is problematic as 
it is well established that highly parameterized models tend to have poor out-of-sample forecasting performance. Moreover, because the probit is nonlinear, the marginal change in the predicitve probabilities are functions of the values of all of the included variables. This means that including irrelevant variables could bias the forecasts. Here, we focus on a modified version of (1), in which not all variables in $X_{t}$ need be included in the model. In particular, define $\gamma$ as an $n \times 1$ vector of zeros and ones, with a one indicating that the corresponding variable in $X_{t}$ should be included in the model. We rewrite (1) to incorporate this variable selection as follows:

$$
\operatorname{Pr}\left[S_{t+h}=1 \mid \rho_{\gamma}, \gamma\right]=\Phi\left(\alpha+X_{\gamma, t}^{\prime} \beta_{\gamma}\right)
$$

where $X_{\gamma, t}, \rho_{\gamma}$, and $\beta_{\gamma}$ contain the elements of $X_{t}, \rho$, and $\beta$ relevant for the variables selected by $\gamma$. As is described in the next subsection, we treat $\gamma$ as unknown, and estimate its value along with the parameters of the model using Bayesian techniques.

\subsection{Estimation}

To estimate the model in (2) we take a Bayesian approach, which has some key advantages for our purposes. For one, uncertainty about which variables should be included in the model - that is uncertainty about $\gamma$ - can be formally incorporated into Bayesian estimation in a straightforward manner. Related to this, the Bayesian framework provides a mechanism, through the posterior predictive density, to obtain forecasts that average over different choices for variable inclusion and the values of unknown parameters.

Bayesian estimation requires priors be placed on the model parameters, $\rho_{\gamma}$, as well as the covariate selection vector, $\gamma$. We specify diffuse, i.i.d., mean-zero normal distributions for the individual parameters collected in $\rho_{\gamma}$ :

$$
p\left(\rho_{\gamma}\right)=N\left(\mathbf{0}_{k_{\gamma}+1}, \sigma^{2} \mathbf{I}_{k_{\gamma}+1}\right) ; \quad \sigma^{2}=10
$$


where $k_{\gamma}=\gamma^{\prime} \gamma$ is the number of covariates selected by $\gamma, \mathbf{0}_{k_{\gamma}+1}$ is a $\left(k_{\gamma}+1\right) \times 1$ vector of zeros, and $\mathbf{I}_{k_{\gamma}+1}$ is the $\left(k_{\gamma}+1\right) \times\left(k_{\gamma}+1\right)$ identity matrix. For $\gamma$, we specify a multinomial distribution defined across the $2^{n}$ different possible choices of $\gamma$. Let $N_{i}=\left(\begin{array}{c}n \\ i\end{array}\right)$ be the number of choices of $\gamma$ for which $k_{\gamma}=i$. The prior probability over $\gamma$ is then:

$$
\operatorname{Pr}(\gamma) \propto \frac{1}{N_{k_{\gamma}}}
$$

This distribution is flat in two dimensions. First, it assigns equal probability to all choices of $\gamma$ that have the same $k_{\gamma}$. In other words, versions of (2) with the same number of covariates will receive equal prior probability. Second, the prior assigns equal cumulative probability to groups of choices for $\gamma$ that imply different numbers of covariates. That is, $\operatorname{Pr}\left(k_{\gamma}=i\right)=\operatorname{Pr}\left(k_{\gamma}=j\right), \quad i, j=0,1, \cdots, n .^{7}$

To implement Bayesian estimation, we employ the Gibbs sampler to obtain draws from the joint posterior distribution, $\pi\left(\rho_{\gamma}, \gamma \mid \mathbf{S}^{t}\right)$, where $\mathbf{S}^{t}=\left[S_{h+1}, \cdots, S_{t}\right]^{\prime}$ represents the observed data. ${ }^{8}$ The Gibbs sampler is facilitated by augmenting the system with a continuous variable $y_{t}$ that is deterministically related to the observed state variable $S_{t}$ (Tanner and Wong (1987)). Define $y_{t}$ as:

$$
y_{t}=\alpha+X_{\gamma, t-h}^{\prime} \beta_{\gamma}+u_{t}
$$

where $u_{t} \sim$ i.i.d. $N(0,1)$. Given $(2)$, the relationship between $y_{t}$ and $S_{t}$ is:

$$
S_{t}=1 \quad \text { if } \quad y_{t} \geq 0
$$

The Gibbs sampler is then implemented in two blocks. In the first, $\rho_{\gamma}$ and $\gamma$ are sampled

\footnotetext{
${ }^{7}$ Note that this prior does not assign equal probability to all possible choices of $\gamma$. While seemingly attractive as a "flat" prior, an equal weights prior would give substantially different prior weight to the number of variables included. For example, if there are 50 possible variables, the cumulative prior probability of all models with 3 variables would be 16 times the cumulative prior probability of all models with 2 variables. ${ }^{8}$ See, for example, Albert and Chib (1993), Gelfand and Smith (1990), Casella and George (1992), and Carter and Kohn (1994).
} 
conditional on $\mathbf{S}^{t}$ and the augmented data $\mathbf{y}^{t}=\left[y_{h+1}, \cdots, y_{t}\right]^{\prime}$, as a draw from the conditional posterior distribution $\pi\left(\rho_{\gamma}, \gamma \mid \mathbf{y}^{t}, \mathbf{S}^{t}\right)$. As $\mathbf{S}^{t}$ is fully determined by $\mathbf{y}^{t}$, this distribution simplifies to $\pi\left(\rho_{\gamma}, \gamma \mid \mathbf{y}^{t}\right)$. In the second, the augmented data $\mathbf{y}^{t}$ is sampled conditional on $\rho_{\gamma}$, $\gamma$, and $\mathbf{S}^{t}$, from the conditional posterior distribution $\pi\left(\mathbf{y}^{t} \mid \rho_{\gamma}, \gamma, \mathbf{S}^{t}\right)$. We now describe each of these blocks in detail:

\section{Sampling $\pi\left(\rho_{\gamma}, \gamma \mid \mathbf{y}^{t}\right)$}

As suggested by Holmes and Held (2006), we jointly sample $\rho_{\gamma}$ and $\gamma$ from:

$$
\pi\left(\rho_{\gamma}, \gamma \mid \mathbf{y}^{t}\right)=\pi_{\rho}\left(\rho_{\gamma} \mid \gamma, \mathbf{y}^{t}\right) \operatorname{Pr}\left(\gamma \mid \mathbf{y}^{t}\right)
$$

by employing a Metropolis step. Given a previous draw of $\rho_{\gamma}$ and $\gamma$, denoted $\left[\rho_{\gamma}^{[g]}, \gamma^{[g]}\right]$, we obtain a candidate for the covariate selection vector, denoted $\gamma^{*}$, by sampling a proposal distribution $q\left(\gamma^{*} \mid \gamma^{[g]}\right)$. Conditional on $\gamma^{*}$, we then obtain a candidate for $\rho_{\gamma}$, denoted $\rho_{\gamma}^{*}$, by sampling from the full conditional posterior density $\pi_{\rho}\left(\rho_{\gamma} \mid \gamma^{*}, \mathbf{y}^{t}\right)$.

The proposal distribution $q\left(\gamma^{*} \mid \gamma^{[g]}\right)$ is set as follows. Conditional on $\gamma^{[g]}$, the candidate covariate selection vector $\gamma^{*}$ is drawn with equal probability from the set of vectors that includes $\gamma^{[g]}$ and all other vectors that alter a single element of $\gamma^{[g]}$ (either from 0 to 1 or 1 to 0 .) In other words, the candidate covariate selection vector will either select the same covariates as $\gamma^{[g]}$, take away one covariate from $\gamma^{[g]}$, or add one covariate to $\gamma^{[g]}$. One notable property of this proposal distribution is that $q\left(\gamma^{*} \mid \gamma^{[g]}\right)$ will equal $q\left(\gamma^{[g]} \mid \gamma^{*}\right)$.

The full conditional distribution for $\rho, \pi_{\rho}\left(\rho_{\gamma} \mid \gamma^{*}, \mathbf{y}^{t}\right)$, is as follows. Define $\mathbf{X}_{\gamma, t-h}=$ $\left[1, X_{\gamma, t-h}^{\prime}\right], t=h+1, \cdots, t$ and let $\mathbf{X}_{\gamma}^{t-h}$ represent the $(t-h) \times\left(k_{\gamma}+1\right)$ matrix of stacked $\mathbf{X}_{\gamma, t-h}$. Then, given the prior distribution in (3), the conditional posterior for $\rho_{\gamma}$ is:

$$
\pi_{\rho}\left(\rho_{\gamma} \mid \gamma^{*}, \mathbf{y}^{t}\right) \sim N\left(\mathbf{m}_{\gamma^{*}}, \mathbf{M}_{\gamma^{*}}\right)
$$

where: 


$$
\begin{gathered}
\mathbf{M}_{\gamma^{*}}=\left(\frac{1}{\sigma^{2}} \mathbf{I}_{k_{\gamma}+1}+\left(\mathbf{X}_{\gamma}^{t-h}\right)^{\prime} \mathbf{X}_{\gamma}^{t-h}\right)^{-1} \\
\mathbf{m}_{\gamma^{*}}=\mathbf{M}_{\gamma^{*}}\left(\left(\mathbf{X}_{\gamma}^{t-h}\right)^{\prime} \mathbf{y}^{t-h}\right)
\end{gathered}
$$

The candidate, $\rho_{\gamma}^{*}$ is then sampled from $N\left(\mathbf{m}_{\gamma^{*}}, \mathbf{M}_{\gamma^{*}}\right)$.

The Metropolis step assigns an acceptance probability $A$ to determine whether or not the candidate will be accepted. Given the $g^{t h}$ draw $\left[\rho_{\gamma}^{[g]}, \gamma^{[g]}\right]$, the $(g+1)^{t h}$ draw is determined by:

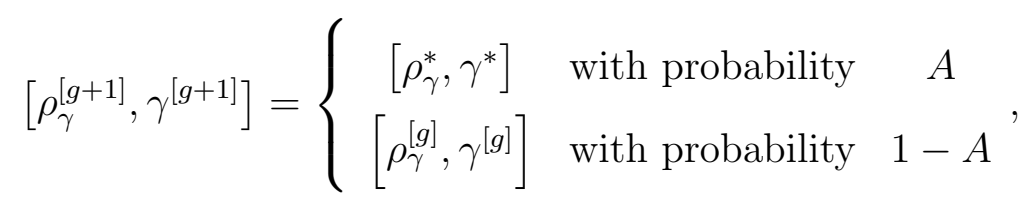

where,

$$
A=\min \left\{1, \frac{\operatorname{Pr}\left(\gamma^{*} \mid \mathbf{y}^{t}\right) \pi_{\rho}\left(\rho_{\gamma}^{*} \mid \gamma^{*}, \mathbf{y}^{t}\right) q\left(\gamma^{[g]} \mid \gamma^{*}\right) \pi_{\rho}\left(\rho_{\gamma}^{[g]} \mid \gamma^{[g]}, \mathbf{y}^{t}\right)}{\operatorname{Pr}\left(\gamma^{[g]} \mid \mathbf{y}^{t}\right) \pi_{\rho}\left(\rho_{\gamma}^{[g]} \mid \gamma^{[g]}, \mathbf{y}^{t}\right) q\left(\gamma^{*} \mid \gamma^{[g]}\right) \pi_{\rho}\left(\rho_{\gamma}^{*} \mid \gamma^{*}, \mathbf{y}^{t}\right)}\right\}
$$

which, given the symmetry of $q(\cdot \mid \cdot)$, simplifies to

$$
A=\min \left\{1, \frac{\operatorname{Pr}\left(\gamma^{*} \mid \mathbf{y}\right)}{\operatorname{Pr}\left(\gamma^{[g]} \mid \mathbf{y}\right)}\right\}
$$

From Bayes' Rule:

$$
\operatorname{Pr}(\gamma \mid \mathbf{y}) \propto f(\mathbf{y} \mid \gamma) \operatorname{Pr}(\gamma)
$$

where $f(\mathbf{y} \mid \gamma)$ is the marginal likelihood for the augmented data, $\mathbf{y}^{t}$, conditional on the choice of variables $\gamma$, and $\operatorname{Pr}(\gamma)$ is the prior distribution over $\gamma$. We can then rewrite the acceptance probability as: 


$$
A=\min \left\{1, \frac{f\left(\mathbf{y}^{t} \mid \gamma^{*}\right) \operatorname{Pr}\left(\gamma^{*}\right)}{f\left(\mathbf{y}^{t} \mid \gamma^{[g]}\right) \operatorname{Pr}\left(\gamma^{[g]}\right)}\right\}
$$

To compute $A$, we must compute $f\left(\mathbf{y}^{t} \mid \gamma\right)$. Given the prior distribution in (3), this is available analytically, as:

$$
f\left(\mathbf{y}^{t} \mid \gamma\right) \sim N\left(0, \Sigma_{\gamma}\right)
$$

where $\Sigma_{\gamma}=I_{t-h}+\sigma^{2} \mathbf{X}_{\gamma}^{t-h}\left(\mathbf{X}_{\gamma}^{t-h}\right)^{\prime}$. Using the equation for the multivariate normal we then have:

$$
A=\min \left\{1, \frac{\left|\Sigma_{\gamma^{[g]}}\right|^{0.5} \exp \left(-0.5\left(\left(\mathbf{y}^{t}\right)^{\prime}\left(\Sigma_{\gamma^{*}}\right)^{-1} \mathbf{y}^{t}\right)\right) \operatorname{Pr}\left(\gamma^{*}\right)}{\left|\Sigma_{\gamma^{*}}\right|^{0.5} \exp \left(-0.5\left(\left(\mathbf{y}^{t}\right)^{\prime}\left(\Sigma_{\gamma^{[g]}}\right)^{-1} \mathbf{y}^{t}\right)\right) \operatorname{Pr}\left(\gamma^{[g]}\right)}\right\} .
$$

Sampling $\pi\left(\mathbf{y} \mid \rho_{\gamma}, \gamma, \mathbf{S}\right)$

Conditional on $\rho_{\gamma}$ and $\gamma, y_{t}$ is normally distributed with mean $\delta_{\gamma, t}$ and unit variance, where:

$$
\delta_{\gamma, t}=\alpha+X_{\gamma, t-h}^{\prime} \beta_{\gamma}
$$

However, the target distribution also conditions on $\mathbf{S}^{t}$, which adds the requirement that the sign of $y_{t}$ must match the realization of $S_{t}$. In this case, $y_{t}$ has a truncated normal density:

$$
y_{t} \sim\left\{\begin{array}{ll}
N\left(\delta_{\gamma, t}, 1\right) I_{\left[y_{t} \geq 0\right]} & \text { if } S_{t}=1 \\
N\left(\delta_{\gamma, t}, 1\right) I_{\left[y_{t}<0\right]} & \text { if } S_{t}=0
\end{array},\right.
$$

where the indicator $I_{[.]}$reflects the direction of the truncation. A draw from $\mathbf{y}^{t}$ is then obtained by drawing $y_{t}$ from the appropriate truncated normal distribution, $t=h+1, \cdots, T$. 
Given arbitrary starting values for $\rho_{\gamma}$ and $\gamma$, the above two sampling steps are iterated

to obtain draws $\left[\rho_{\gamma}^{[g]}, \gamma^{[g]}\right]$, for $g=1, \cdots, G$. Following a suitably large initialization period, these draws will be from the joint posterior distribution, $\pi\left(\rho_{\gamma}, \gamma \mid \mathbf{S}^{t}\right)$. In all estimations reported below, we sample 20,000 initialization draws to achieve convergence. Results are then based on an additional set of $G=20,000$ draws. We verified the adequacy of the initialization period by ensuring that results from two different runs of the Gibbs Sampler with dispersed sets of starting values were similar.

\subsection{Construction of Forecasts and Forecast Evaluation}

To forecast the business cycle phase in period $t+h$, we use the posterior predictive distribution:

$$
\operatorname{Pr}\left[S_{t+h}=1 \mid \mathbf{S}^{t}\right]
$$

Although the posterior predictive distribution is not available analytically, we can simulate from (7) as follows. The posterior predictive distribution is factored as:

$$
\operatorname{Pr}\left[S_{t+h}=1 \mid \mathbf{S}^{t}\right]=\int_{\rho_{\gamma}} \int_{\gamma} \operatorname{Pr}\left[S_{t+h}=1 \mid \rho_{\gamma}, \gamma, \mathbf{S}^{t}\right] \pi\left(\rho_{\gamma}, \gamma \mid \mathbf{S}^{t}\right) d_{\gamma} d_{\rho_{\gamma}}
$$

Equation (8) suggests a Monte Carlo integration approach to calculate the posterior predictive distribution. Specifically, given a draw $\left[\rho_{\gamma}^{[g]}, \gamma^{[g]}\right]$ from $\pi\left(\rho_{\gamma}, \gamma \mid \mathbf{S}^{t}\right)$ we simulate a value of $S_{t+h}$, denoted $S_{t+h}{ }^{[g]}$, from:

$$
\operatorname{Pr}\left[S_{t+h}=1 \mid \rho_{\gamma}, \gamma, \mathbf{S}^{t}\right]=\operatorname{Pr}\left[S_{t+h}=1 \mid \rho_{\gamma}, \gamma,\right]=\Phi\left(\alpha+X_{\gamma, t}^{\prime} \beta_{\gamma}\right)
$$

where the validity of the first equality sign comes from the fact that, given the model in (2) and the model parameters, the observed data $\mathbf{S}^{t}$ are not informative for the distribution of $S_{t+h}$. The simulated value $S_{t+h}^{[g]}$ is a draw from the posterior predictive distribution, 
$\operatorname{Pr}\left[S_{t+h} \mid \mathbf{S}^{t}\right]$, and it follows that:

$$
\lim _{G \rightarrow \infty} \frac{1}{G} \sum_{g=1}^{G}\left(S_{t+h}{ }^{[g]}\right)=\operatorname{Pr}\left[S_{t+h}=1 \mid \mathbf{S}^{t}\right]
$$

Thus, we can construct a simulation consistent estimate of the posterior predictive density $\operatorname{Pr}\left[S_{t+h}=1 \mid \mathbf{S}^{t}\right]$, which will serve as our (point) forecast of $S_{t+h}$. In the following, we refer to this forecast as $\widehat{S}_{t+h}$. It is worth emphasizing that $\widehat{S}_{t+h}$ is not conditional on model parameters or the choice of which variables to include in the model. These sources of uncertainty have been integrated, over their respective posterior distributions, out of the prediction. Note that the integration over the posterior distribution for $\gamma$ gives $\widehat{S}_{t+h}$ the interpretation of a BMA prediction.

To evaluate $\widehat{S}_{t+h}$, we consider a variety of forecast evaluation metrics. The first is the Brier (1950) quadratic probability score $(Q P S)$, which is a probability analog of mean squared error:

$$
Q P S=\frac{2}{\tau_{2}-\tau_{1}} \sum_{t+h=\tau_{1}+1}^{\tau_{2}}\left(\widehat{S}_{t+h}-S_{t+h}\right)^{2}
$$

where $\tau_{1}$ and $\tau_{2}$ are chosen to cover the period over which $\widehat{S}_{t+h}$ is being evaluated. The $Q P S$ ranges from 0 to 2 , with 0 indicating perfect forecast accuracy.

While the $Q P S$ is a standard metric for the evaluation of forecasts of binary variables, it does not directly evaluate $\widehat{S}_{t+h}$ as a classifier of $S_{t+h}$. Specifically, the $Q P S$ is focused on the distance between $\widehat{S}_{t+h}$ and $S_{t+h}$, rather than on the ability of $\widehat{S}_{t+h}$ to classify $S_{t+h}$ accurately. This distinction is meaningful, as it is simple to construct examples where a forecasting model with perfect classification ability has an inferior $Q P S$ to a model with less than perfect classification ability. ${ }^{9}$ Thus, we also consider two forecast evaluation metrics that directly assess classification ability. The first is the correspondence, defined as the proportion of months for which $\widehat{S}_{t+h}$ correctly indicates the NBER business cycle phase. ${ }^{9}$ See, e.g., Hand and Vinciotti (2003). 
The correspondence (CSP) is given by:

$$
C S P=\frac{1}{\tau_{2}-\tau_{1}} \sum_{t+h=\tau_{1}+1}^{\tau_{2}}\left(I_{\left[\widehat{S}_{t+h} \geq c\right]} S_{t+h}+\left(1-I_{\left[\widehat{S}_{t+h} \geq c\right]}\right)\left(1-S_{t+h}\right)\right)
$$

where $c$ is a threshold point such that when $\widehat{S}_{t+h}$ exceeds $c$ we classify a month as a recession, and $I_{\left[\widehat{S}_{t+h} \geq c\right]} \in\{0,1\}$ is an indicator function denoting the predicted business cycle phase. In the results presented below, we set $c=0.5$. Also, rather than focus directly on the correspondence, we present a measure of "excess correspondence" (XCSP) that shows the increase in the CSP above the correspondence produced by a simple classifier that sets $\widehat{S}_{t+h}=1$ with probability equal to the sample mean of $\mathbf{S}^{t}$, and sets $\widehat{S}_{t+h}=0$ otherwise.

The correspondence requires that we set a value of $c$, and while $c=0.5$ is a natural threshold for a probability in terms of likelihood, it could easily distort the performance of a classifier for which an alternative threshold was appropriate. Thus, for our final forecast evaluation metric we follow Berge and Jordá (2011) and consider the area under the receiver operating characteristic, or ROC, curve. The ROC curve summarizes the classification performance of a continuous index, such as $\widehat{S}_{t+h}$, by plotting the the proportion of true positives against the proportion of false positives, where each is calculated for the range of possible thresholds as $c$ is varied from 1 to 0 (moving from left to right in the plot.) The area under this curve, or $A U C$, is a summary statistic describing classification performance, with a perfect classifier having an $A U C=1$ and a coin-flip classifier having $A U C=0.5$. Unlike the CSP statistic, the $A U C$ is not dependent on a particular value of $c$. An estimate of the $A U C$ is given by:

$$
\widehat{A U C}=\frac{1}{n_{0} n_{1}} \sum_{j=1}^{n_{0}} \sum_{i=1}^{n_{1}}\left(I_{\left[\widehat{S}_{i}^{1}>\widehat{S}_{j}^{0}\right]}\right)
$$

where $\widehat{S}_{i}^{1}, i=1, \cdots, n_{1}$ are the values of $\widehat{S}_{t+h}$ for the $n_{1}$ out-of-sample periods for which $S_{t+h}=1$ and $\widehat{S}_{j}^{0}, j=1, \cdots, n_{0}$ are the values of $\widehat{S}_{t+h}$ for the $n_{0}$ out-of-sample periods for which $S_{t+h}=0$. 


\section{Data}

For the dependent variable in our estimation, $S_{t}$, we use the monthly chronology of recessions and expansion dates provided by the NBER's Business Cycle Dating Committee. Our predictor variables consist of both national- and state-level variables, all of which are sampled at the monthly frequency. For the national-level variables, we include a measure of the term spread, the federal funds rate, and the return on the S\&P 500 stock market index. Each of these variables have been shown to help predict recessions at various horizons in the existing literature. ${ }^{10}$ We also include two direct measure of aggregate economic activity, namely payroll employment growth and industrial production growth. Such variables are of obvious interest for nowcasting, and have also been found helpful for short-horizon business cycle phase forecasting (Estrella and Mishkin (1998).)

In addition to this standard set of national-level variables, we also include a measure of state-level economic activity, namely state-level payroll employment growth. We choose payroll employment growth as the measure of state-level economic activity for two reasons. First, we are interested in relatively high frequency monitoring of business cycle phases. Payroll employment is the broadest measure of state-level economic activity that is sampled at a monthly frequency. ${ }^{11}$ Second, as compared to other monthly measures of state-level activity, such as retail sales, payroll employment is released quickly, roughly three weeks following the end of the month. This timeliness makes payroll employment attractive for nowcasting and forecasting.

The specific transformations applied to each variable are as follows. The term spread is measured as the difference between the monthly averages of the 10-year Treasury bond and the 3-month Treasury bill, while the Federal Funds rate is measured as its monthly average value. The S\&P 500 return is the three month growth rate of the S\&P 500 index. Industrial production and national- and state-level payroll employment growth are the three

\footnotetext{
${ }^{10}$ See e.g., Estrella and Mishkin (1998), Wright (2006), King et al. (2007), and Berge (2013).

${ }^{11}$ The most comprehensive measure of state-level economic activity, Gross State Product, is only released annually.
} 
month growth rates of the underlying levels of these variables. ${ }^{12}$ We focus on three month growth rates in order to smooth month-to-month variability in these series that is unrelated to shifts in business cycle phases. We have also generated results using one month growth rates, and these were uniformly inferior for models using only national-level data as well as models using national and state-level data. However, the relative forecasting advantage of using state-level data remained when using one month growth rates.

All data series were collected over the period from January 1960 to August 2013. After constructing growth rates and adjusting for the maximum forecast horizon considered, the full sample period for $S_{t+h}$ covers from August 1960 to August 2013. Over this period there are eight NBER defined recessions, and approximately $14 \%$ of the monthly observations are recession months.

In our primary analysis, we use variables collected as of the September 2013 vintage. Thus, for variables that are revised, which is the case for the economic activity measures in our sample, we use ex-post revised data in our out-of-sample forecasting experiments rather than the vintage of data that would have been available to a forecaster in real time. We make this choice due to difficulties with obtaining long histories for state-level payroll employment at a substantial number of vintages over our out-of-sample forecasting period. However, as a robustness check, we additionally report results of an out-of-sample forecasting experiment over a shorter time period for which we were able to obtain real-time data.

\section{Results}

This section presents results to assess the out-of-sample forecast performance of the probit model augmented with state-level data. The state-level employment data we consider is likely a coincident indicator of state-level business cycles. As discussed previously, it is possible that state-level employment data might lead the national business cycle phase at long horizons, which would be true if there was a systematic long lead of certain state-

\footnotetext{
${ }^{12}$ Where relevant, the underlying data are seasonally-adjusted.
} 
level business cycle phases over the national business cycle phase. However, our out-ofsample forecast analysis suggests that forecast improvements coming from use of state-level data exist only at very short horizons. This suggests that the primary benefit of the state level data comes from exploitable short leads of state-level business cycles over the national business cycle, or information provided by certain states that a recession has already begun. To highlight these short-horizon forecast improvements here, we present results only for four short forecast horizons, consisting of $h=0,1,2,3$ months ahead. Again, improved shorthorizon forecasts are of substantial interest, since definitive classification of new business cycle phases have historically only been available with a substantial lag.

In all cases, we assume that the information used to predict $S_{t+h}$ consists of the information available at the end of month $t$. For the financial variables in our data set, this includes the values of these variables measured for month $t$. For each of the real-activity measures in the data set, both at the national- and state-level, this includes the values of these variables measured for month $t-1$. As an example, a one-month-ahead forecast in our context refers to a prediction of $S_{t+1}$ formed using financial variables measured for month $t$ and economic activity variables measured for month $t-1$. A "nowcast" refers to the case of $h=0$, and corresponds to a prediction of the business cycle phase in month $t$, formed using data available at the end of month $t$, some of which is data measured for month $t$ and some of which is measured for month $t-1$.

The out-of-sample period extends from January 1979 to August 2013. The initial estimation period is from August 1960 to December 1978, and the first out-of-sample forecast for $h=0$ and $h=1$ is January 1979, for $h=2$ is February 1979, and for $h=3$ is March 1979. The last out-of-sample forecast for all horizons is August 2013. After each out-of-sample forecast is produced, the estimation sample is extended by one month, and the model reestimated. The out-of-sample period includes 5 NBER-defined recessions, accounting for approximately $14 \%$ of the 416 months over this period.

Table 1 presents the forecast evaluation metrics for two alternative models. The first uses 
only national-level predictors. This model will serve as our baseline with which to compare the second model, which includes both national- and state-level predictors. These metrics tell a consistent story: the model that includes state-level data produces better forecasts than the baseline model for very short horizon forecasts, namely nowcasts $(h=0)$ and one-step ahead forecasts $(h=1$.) At these horizons, the $Q P S$ is lower, while the XCSP and AUC are higher, for the model including state-level data. For the $Q P S$ and $X C S P$, we further test the null hypothesis of equal forecast accuracy using the Diebold-Mariano-West (DMW) test, and find that this can be rejected for $X C S P$ when $h=0$ and for both metrics when $h=1 .{ }^{13}$ The forecast improvements as measured by several metrics are quantitatively large. As one example, the XCSP is 3 percentage points higher for the model including state-level data when $h=1$, meaning that approximately 12 more months were correctly classified by the model that includes state-level data. For longer horizons, the inclusion of state-level data appears less helpful. For $h=2$ and $h=3$, the forecast metrics are very similar for both the baseline model and the model that includes state-level data.

We next investigate whether the improvements generated by the inclusion of state-level data are symmetric across business cycle phases. Table 2 presents the forecast evaluation metrics computed separately for expansion vs. recession months in the out-of-sample period. ${ }^{14}$ These results demonstrate that the forecast improvements generated by the inclusion of state-level data in the out-of-sample period are concentrated in recession months. In particular, the forecast evaluation metrics computed for expansions are generally similar for the baseline model and the model that includes state-level data, with which model has better performance differing across horizon and forecast evaluation metric. However, when we focus on recession months, there is a clear benefit from incorporating state-level data for short-horizon forecasts. For $h=0$ and $h=1$, the QPS is reduced by $50 \%$ to $60 \%$ during recession months. The XCSP improvements are approximately 25 percentage points at

\footnotetext{
${ }^{13}$ See Diebold and Mariano (1995) and West (1996).

${ }^{14}$ We do not report the AUC in Table 2 as it is only defined where there are instances of both classes in the sample considered.
} 
these horizons, meaning that a quarter of the recession months over the sample period are correctly classified by the model that includes state-level data, but not by the model that includes only national-level data. The $Q P S$ and $X C S P$ improvements are highly statistically significant based on the DMW test. There are again no clear improvements from the addition of state-level data at longer horizons.

To provide an example from a specific recession, Table 3 presents the out-of-sample forecasts, $\widehat{S}_{t+h}$, for the $h=1$ case around the 2008-2009 recession. Beginning with the model that includes only national-level variables, $\widehat{S}_{t+1}$ does not cross $50 \%$ probability of recession until August 2008, eight months following the beginning of the NBER-defined recession. For the $h=1$ horizon, this forecast would have been available at the end of July 2008. This is consistent with the considerable uncertainty that persisted well into 2008 about whether the economy had entered a recession phase. For example, the NBER did not announce the December 2007 peak until December 1, 2008. Also, as discussed in Hamilton (2011), statistical models designed to track business cycle turning points using national-level data did not send a definitive signal that the recession had begun until mid-to-late 2008. However, Table 3 also reveals that incorporating state-level data would have provided a much quicker signal of the beginning of this recession. Specifically, $\widehat{S}_{t}$ moved above $50 \%$ probability of recession for March 2008, where this forecast would have been available as of the end of February 2008, an impressive five month improvement over the model using only national-level data. Notably, both models produce accurate one-month ahead forecasts of the end of the 2008-2009 recession.

We next evaluate which variables are deemed as important predictors in the BMA procedure. Table 4 reports the posterior inclusion probabilities for the model that includes both national- and state-level data, averaged over the recursive estimations conducted to construct the out-of-sample forecasts. The table provides these inclusion probabilities for all of the national-level variables. The state-level variables will be discussed separately below. Of the national-level variables, the S\&P 500 return is a robust predictor across all forecast 
horizons, with average inclusion probabilities close to 100\%. The Federal Funds rate also has average inclusion probabilities above $50 \%$ for several forecast horizons. The term spread becomes a more robust predictor as the forecast horizon lengthens, consistent with the finding of the existing literature that this variable is useful for long-horizon business cycle phase predictions. Interestingly, neither aggregate employment or aggregate industrial production growth have average inclusion probabilities above $50 \%$ for most forecast horizons, the single exception being industrial production growth when $h=0$. These variables have very high average inclusion probabilities when state-level data is not included (not reported), implying the importance of these aggregate level variables is substantially diminished by the inclusion of state-level data.

The final row of 4 shows the posterior median for the number of variables in the model, averaged across the recursive estimations. For the $h=0$ and $h=1$ horizon these values are large, at greater than 15 variables. This suggests that there are a substantial number of state-level variables that influence the forecast. To investigate which state level variables these are, Figure 1 maps the states with ranges of inclusion probability. We focus on the $h=0$ and $h=1$ forecast horizons, where the forecast improvements from the addition of state level data are concentrated. These maps show that while there are few states with very high average inclusion probabilities (darker shading), there are many states with average inclusion probabilities in the 20\%-60\% range, meaning a large number of states influence the BMA forecast. These probabilities also indicate significant model uncertainty regarding which state-level variables should be included in the model. One likely reason for this uncertainty is significant correlation between the state-level employment growth variables. This uncertainty again highlights the potential importance of the BMA approach we take to select predictors and incorporate uncertainty about this selection.

Given this potential importance, we next present results meant to evaluate whether the BMA predictor selection algorithm is a significant factor for the out-of-sample forecast improvements generated with the addition of state-level data. Specifically, Table 5 reports the 
forecast evaluation metrics for out-of-sample forecasts produced from a model in which all national- and state-level variables are always included. As the forecasting improvements from adding state-level data were concentrated in short-horizon forecasts of recession months, we focus on the forecast evaluation metrics computed for nowcasts $(h=0)$ and one-month-ahead forecasts $(h=1)$ of recession months over the out-of-sample period. By comparing Table 5 to the bottom panel of Table 2, we can gauge the value added of using the BMA predictor selection algorithm to construct forecasts, vs. simply including all possible variables. Indeed, this comparison shows a deterioration in the out-of-sample forecast performance from conditioning on a model that includes all possible variables rather than using BMA. Notably however, the model with all variables included is still preferred to the model that doesn't include state-level data.

Finally, as was discussed in Section 3 above, our out-of-sample forecasts are constructed using ex-post revised data for the predictors taken from the September 2013 vintage for each series. In Table 6 we evaluate the robustness of the out-of-sample forecasting results when we instead use "real-time" data of vintages that would have been available to a forecaster in real time. Due to difficulties with obtaining long histories for state-level payroll employment at a substantial number of vintages, we focus on a shorter out-of-sample period running from July 2007 to June 2011, which includes the most recent NBER-defined recession. As forecast improvements from the addition of state-level data were primarily at short horizons, we focus on one month ahead forecasts.

Table 6 demonstrates that our primary conclusions from the longer out-of-sample period using ex-post data are confirmed for the shorter out-of-sample period using real-time data. In particular, there is a general improvement in the forecast evaluation metrics computed for recession months from the addition of state-level data. As an example, the XCSP is 17 percentage points higher when state-level data is included, which corresponds to roughly 3 more recession months during the 2008-2009 recession being correctly classified. Also, as before, there is no apparent improvement from the addition of state-level data for one-month 
ahead predictions of expansion phases.

\section{Conclusion}

A large literature has investigated the predictive content of variables measured at the national level, such as aggregate employment and output growth, for forecasting U.S. business cycle phases (expansions and recessions.) Motivated by recent studies showing differences in the timing of business cycle phases in nationally aggregated data from those for geographically disaggregated data, we investigate the information contained in state-level employment growth for forecasting national business cycle phases. We use as a baseline a probit model to explain NBER-defined business cycle phases, where the conditioning information consists of national-level economic activity and financial variables. We then add to this model state-level employment growth. To avoid issues associated with overparameterization of forecasting models, we use a Bayesian model averaging procedure to construct forecasts.

Using a variety of forecast evaluation metrics, we find that adding state-level employment growth improves nowcasts and short-horizon forecasts of the NBER business cycle phase over a model that uses data measured at the national-level only. The gains in forecasting accuracy are concentrated during months of recession, and are large and statistically significant. Pos-

terior inclusion probabilities indicate substantial uncertainty regarding which states belong in the model, highlighting the importance of the Bayesian model averaging approach. 


\section{References}

Albert, J. H. and S. Chib (1993). Bayesian analysis of binary and polychotomous response data. Journal of the American Statistical Association 88(422), 669-679.

Berge, T. (2013). Predicting recessions with leading indicators: Model averaging and selection over the business cycle. Federal Reserve Bank of Kansas City Working Paper No. $13-05$.

Berge, T. and O. Jordá (2011). The classification of economic activity into expansions and recessions. American Economic Journal: Macroeconomics 3(2), 246-277.

Brier, G. W. (1950). Verification of forecasts expressed in terms of probability. Monthly Weather Review 75 (January), 1-3.

Burns, A. F. and W. C. Mitchell (1946). Measuring business cycles. New York: National Bureau of Economic Research. ID: 169122.

Carter, C. K. and R. Kohn (1994). On gibbs sampling for state space models. Biometrika 81(3), 541-553. ID: 476895942.

Casella, G. and E. I. George (1992). Explaining the gibbs sampler. American Statistician 46(3), 167-174. ID: 481283991.

Chauvet, M. and J. Piger (2008). A comparison of the real-time performance of business cycle dating methods. Journal of Business and Economic Statistics 26(1), 42-49.

Diebold, F. X. and R. S. Mariano (1995). Comparing predictive accuracy. Journal of Business and Economic Statistics 13, 253-265.

Diebold, F. X. and G. D. Rudebusch (1991). Forecasting output with the composite leading index: A real-time analysis. Journal of the American Statistical Association 86, 603-610. 
Estrella, A. (1997). A new measure of fit for equations with dichotomous dependent variables. Journal of Business and Economic Statistics 16(2), 198-205.

Estrella, A. and F. S. Mishkin (1998). Predicting u.s. recessions: Financial variables as leading indicators. The Review of Economics and Statistics 80(1), 45-61.

Gelfand, A. E. and A. F. M. Smith (1990). Sampling-based approaches to calculating marginal densities. Journal of the American Statistical Association 85(410), 398-409.

Hamilton, J. D. (1989). A new approach to the economic analysis of nonstationary time series and the business cycle. Econometrica 57(2), 357-384. ID: 480697643.

Hamilton, J. D. (2011). Calling recessions in real time. International Journal of Forecasting $27(4), 1006-1026$.

Hamilton, J. D. and M. T. Owyang (2011). The propagation of regional recessions. NBER working paper no. 16657.

Hand, D. J. and V. Vinciotti (2003). Local versus global models for classification problems: Fitting models where it matters. The American Statistician 57, 124-131.

Hernandez-Murillo, R. and M. T. Owyang (2006). The information content of regional employment data for forecasting aggregate conditions. Economic Letters 90(3), 335-339.

Holmes, C. C. and L. Held (2006). Bayesian auxiliary variable models for binary and multinomial regression. Bayesian Analysis 1(1), 145-168.

Katayama, M. (2008). Improving recession probability forecasts in the u.s. economy. Working Paper.

Kauppi, H. and P. Saikkonen (2008). Predicting u.s. recessions with dynamic binary response models. The Review of Economics and Statistics 90(4), 777-791. 
King, T. B., A. T. Levin, and R. Perli (2007). Financial market perceptions of recession risk. Federal Reserve Board Finance and Economics Discussion Series working paper no. $2007-57$.

Mitchell, W. C. (1927). Business Cycles: The Problem and its Setting. New York: National Bureau of Economic Research.

Owyang, M. T., J. Piger, and H. J. Wall (2005). Business cycle phases in u.s. states. Review of Economics and Statistics 87(4), 604-616.

Rudebusch, G. D. and J. C. Williams (1991). Forecasting recessions: The puzzle of the enduring power of the yield curve. Journal of Business and Economic Statistics 27(4), $492-503$.

Tanner, M. A. and W. H. Wong (1987). The calculation of posterior distributions by data augmentation. Journal of the American Statistical Association 82(398), 528-540.

West, K. D. (1996). Asymptotic inference about predictive ability. Econometrica 64, $1067-$ 1084 .

Wright, J. H. (2006). The yield curve and predicting recessions. Federal Reserve Board Finance and Economics Discussion Series working paper no. 2006-07. 
Figure 1

Average Predictor Inclusion Probabilities for Recursive Estimations
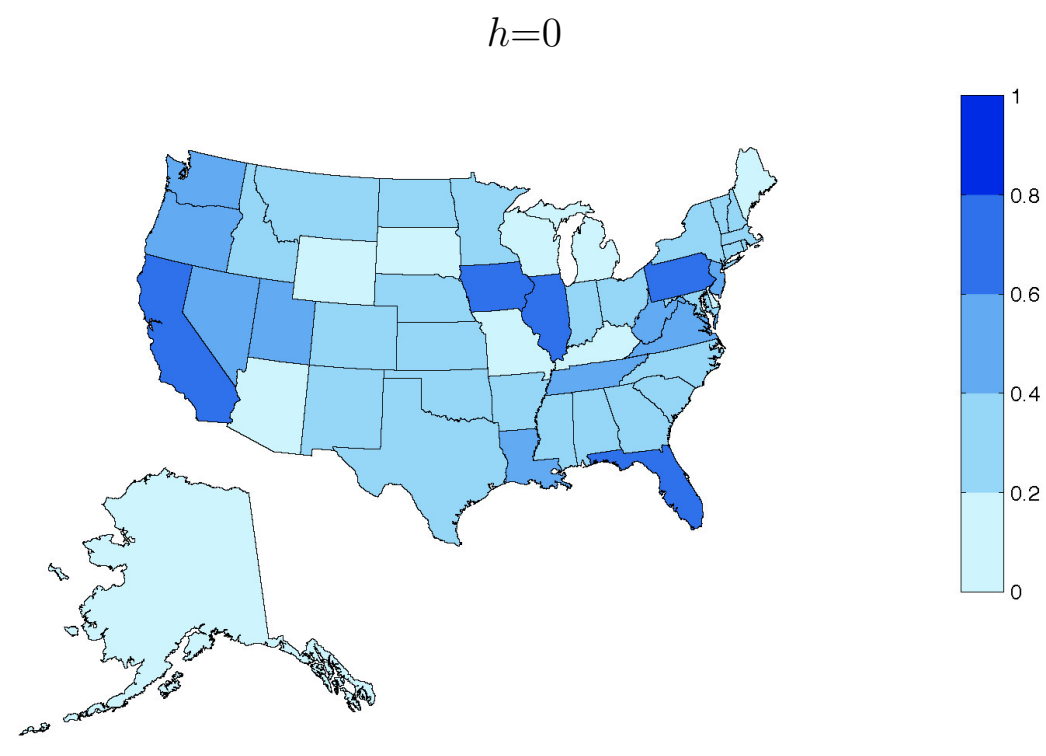

$$
h=1
$$
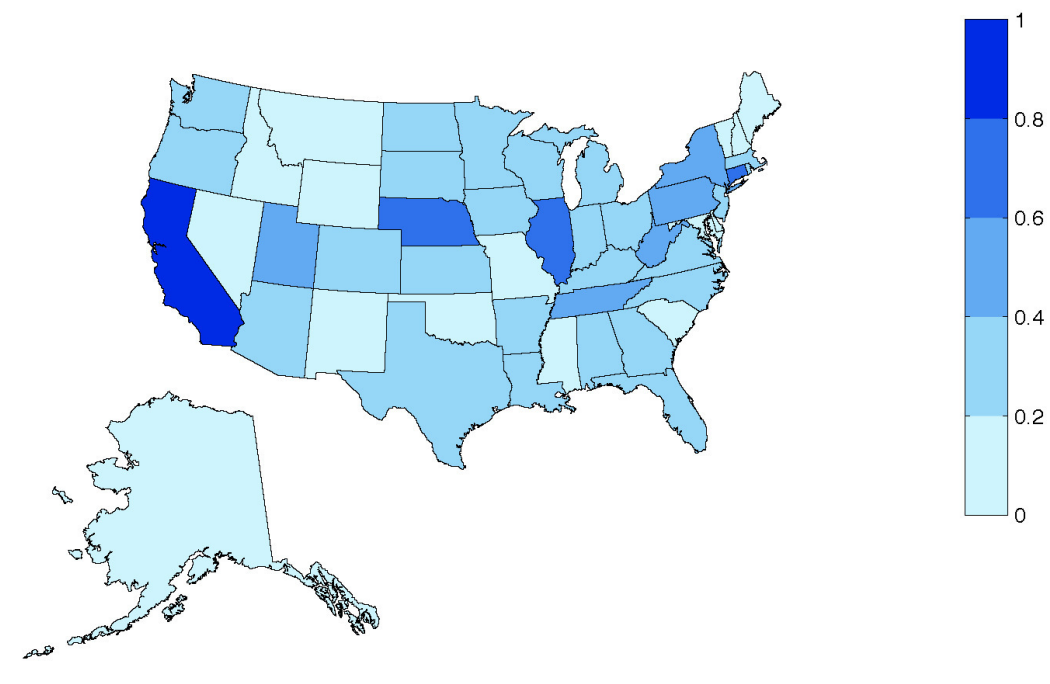

Notes: These maps indicate the average posterior probability that state-level employment variables are included in the model given by (2), where averaging is across the multiple recursive estimations beginning over the period August 1960-December 1978, and ending with the period August 1960-mid 2013, with the exact ending month dependent on the forecasting horizon. 
Table 1

Forecast Evaluation Metrics

\begin{tabular}{|c|c|c|c|c|c|c|}
\hline \multirow[b]{2}{*}{ Forecast Horizon } & \multicolumn{3}{|c|}{$\begin{array}{l}\text { National-Level } \\
\text { Predictors }\end{array}$} & \multicolumn{3}{|c|}{$\begin{array}{c}\text { National and } \\
\text { State-Level Predictors }\end{array}$} \\
\hline & $Q P S$ & $X C S P$ & $A U C$ & $Q P S$ & $X C S P$ & $A U C$ \\
\hline$h=0$ & 0.10 & 0.16 & 0.96 & 0.08 & $0.18^{*}$ & 0.98 \\
\hline$h=1$ & 0.12 & 0.15 & 0.95 & $0.08^{* *}$ & $0.18^{* * *}$ & 0.97 \\
\hline$h=2$ & 0.14 & 0.14 & 0.93 & 0.15 & 0.14 & 0.93 \\
\hline$h=3$ & 0.15 & 0.14 & 0.92 & 0.18 & 0.13 & 0.92 \\
\hline
\end{tabular}

Notes: This table holds the forecast evaluation metrics defined in Section 2.3 constructed for out-of-sample forecasts of the business cycle phase (expansion or recession) produced over the period January 1979 to August 2013. The out-of-sample predictions are constructed from the posterior predictive density of two versions of the model in (2), which differ on the inclusion of state-level predictors. For the QPS and XCSP evaluation metrics, *, **, and $* * *$ indicate a rejection of the null hypothesis of equal forecast accuracy at the $0.10,0.05$ and 0.01 significance level respectively. 
Table 2

Forecast Evaluation Metrics - Expansion vs. Recession Months

\begin{tabular}{|c|c|c|c|c|}
\hline \multirow[b]{2}{*}{ Forecast Horizon } & \multicolumn{2}{|c|}{$\begin{array}{c}\text { National-Level } \\
\text { Predictors }\end{array}$} & \multicolumn{2}{|c|}{$\begin{array}{c}\text { National and } \\
\text { State-Level Predictors }\end{array}$} \\
\hline & $Q P S$ & $X C S P$ & $Q P S$ & $X C S P$ \\
\hline \multicolumn{5}{|l|}{ Expansion Months } \\
\hline$h=0$ & 0.06 & 0.10 & 0.07 & 0.09 \\
\hline$h=1$ & 0.07 & 0.09 & 0.06 & 0.10 \\
\hline$h=2$ & 0.08 & 0.09 & 0.09 & 0.09 \\
\hline$h=3$ & 0.07 & 0.10 & 0.09 & 0.09 \\
\hline \multicolumn{5}{|l|}{ Recession Months } \\
\hline$h=0$ & 0.41 & 0.54 & $0.15^{* * *}$ & $0.78^{* * *}$ \\
\hline$h=1$ & 0.47 & 0.51 & $0.23^{* * *}$ & $0.74^{* * *}$ \\
\hline$h=2$ & 0.51 & 0.47 & 0.51 & 0.47 \\
\hline$h=3$ & 0.64 & 0.40 & 0.71 & 0.38 \\
\hline
\end{tabular}

Notes: This table holds the forecast evaluation metrics defined in Section 2.3 constructed for out-of-sample forecasts of the business cycle phase (expansion or recession) produced separately for NBER defined expansion and recession months over the period January 1979 to August 2013. The out-of-sample predictions are constructed from the posterior predictive density of two versions of the model in (2), which differ on the inclusion of state-level predictors. For the QPS and XCSP evaluation metrics, $*, * *$, and $* * *$ indicate a rejection of the null hypothesis of equal forecast accuracy at the 0.10, 0.05 and 0.01 significance level respectively. 
Table 3

One-Month Ahead Forecasts: 2008-2009 Recession

\begin{tabular}{cccc} 
Date & $\begin{array}{c}\text { NBER Recession } \\
\text { Indicator }\end{array}$ & $\begin{array}{c}\text { National-Level } \\
\text { Predictors }\end{array}$ & $\begin{array}{c}\text { National and State- } \\
\text { Level Predictors }\end{array}$ \\
\hline November 2007 & 0 & 0.08 & 0.05 \\
December 2007 & 0 & 0.08 & 0.02 \\
January 2008 & 1 & 0.06 & 0.00 \\
February 2008 & 1 & 0.36 & 0.38 \\
March 2008 & 1 & 0.19 & $\mathbf{0 . 6 1}$ \\
April 2008 & 1 & 0.42 & $\mathbf{0 . 9 0}$ \\
May 2008 & 1 & 0.16 & $\mathbf{0 . 6 6}$ \\
June 2008 & 1 & 0.15 & 0.10 \\
July 2008 & 1 & 0.30 & $\mathbf{0 . 9 2}$ \\
August 2008 & 1 & $\mathbf{0 . 8 0}$ & $\mathbf{1 . 0 0}$ \\
September 2008 & 1 & $\mathbf{0 . 7 6}$ & $\mathbf{1 . 0 0}$ \\
October 2008 & 1 & $\mathbf{0 . 8 8}$ & $\mathbf{1 . 0 0}$ \\
November 2008 & 1 & $\mathbf{1 . 0 0}$ & $\mathbf{1 . 0 0}$ \\
December 2008 & 1 & $\mathbf{1 . 0 0}$ & $\mathbf{1 . 0 0}$ \\
January 2009 & 1 & $\mathbf{1 . 0 0}$ & $\mathbf{1 . 0 0}$ \\
February 2009 & 1 & $\mathbf{1 . 0 0}$ & $\mathbf{1 . 0 0}$ \\
March 2009 & 1 & $\mathbf{1 . 0 0}$ & $\mathbf{1 . 0 0}$ \\
April 2009 & 1 & $\mathbf{1 . 0 0}$ & $\mathbf{0 . 9 0}$ \\
May 2009 & 1 & $\mathbf{1 . 0 0}$ & 0.20 \\
June 2009 & 1 & $\mathbf{0 . 6 6}$ & 0.26 \\
July 2009 & 0 & 0.17 & \\
August 2009 & 0 & 0.35 & \\
& & &
\end{tabular}

Notes: This table holds the one-month ahead out-of-sample forecasts of the business cycle phase (expansion and recession) around the 2008-2009 NBER-defined recession. The forecasts are constructed from two versions of the model in (2), which differ on the inclusion of state-level predictors. 
Table 4

Average Predictor Inclusion Probabilities for Recursive Estimations

\begin{tabular}{lcccc} 
& $h=0$ & $h=1$ & $h=2$ & $h=3$ \\
\hline National-Level Predictors & & & & \\
& & & & \\
Federal Funds Rate & 0.71 & 0.65 & 0.59 & 0.20 \\
S\&P 500 Return & 0.97 & 1.00 & 0.98 & 1.00 \\
Term Spread & 0.37 & 0.64 & 0.74 & 0.98 \\
Employment Growth & 0.25 & 0.39 & 0.15 & 0.08 \\
Industrial Production Growth & 0.66 & 0.39 & 0.08 & 0.04 \\
& & & & \\
Number of Variables & $\mathbf{1 6 . 8 8}$ & $\mathbf{1 5 . 8 3}$ & $\mathbf{1 0 . 8 2}$ & $\mathbf{9 . 7 8}$ \\
\hline
\end{tabular}

Notes: This table holds the average posterior probability that the national-level variables are included in the model given by (2), when both national and state-level variables are included in the list of potential variables. Averaging is across the multiple recursive estimations beginning over the period August 1960-December 1978, and ending with the period August 1960-mid 2013, with the exact ending month dependent on the forecasting horizon. 
Table 5

Forecast Evaluation Metrics - Recession Months

All National- and State-Level Predictors Included

\begin{tabular}{ccc} 
Forecast Horizon & QPS & XCSP \\
\hline$h=0$ & 0.23 & 0.68 \\
$h=1$ & 0.20 & 0.72 \\
\hline
\end{tabular}

Notes: This table holds the forecast evaluation metrics defined in Section 2.3 constructed for out-of-sample forecasts of the business cycle phase (expansion or recession) computed separately for NBER defined recession months over the period January 1979 to August 2013. The out-of-sample predictions are constructed from the posterior predictive density of the model in (2), where all possible covariates, both national- and state-level, are included. 
Table 6

Forecast Evaluation Metrics with "Real-Time" Data

\begin{tabular}{|c|c|c|c|c|}
\hline \multirow[b]{2}{*}{ Forecast Horizon } & \multicolumn{2}{|c|}{$\begin{array}{l}\text { National-Level } \\
\text { Predictors }\end{array}$} & \multicolumn{2}{|c|}{$\begin{array}{c}\text { National and } \\
\text { State-Level Predictors }\end{array}$} \\
\hline & $Q P S$ & $X C S P$ & $Q P S$ & $X C S P$ \\
\hline \multicolumn{5}{|l|}{ All Months } \\
\hline$h=1$ & 0.24 & 0.04 & 0.24 & 0.08 \\
\hline \multicolumn{5}{|l|}{ Expansion Months } \\
\hline$h=1$ & 0.01 & 0.14 & 0.04 & 0.11 \\
\hline \multicolumn{5}{|l|}{ Recession Months } \\
\hline$h=1$ & 0.64 & 0.37 & 0.57 & 0.54 \\
\hline
\end{tabular}

Notes: This table holds the forecast evaluation metrics defined in Section 2.3 constructed for out-of-sample forecasts of the business cycle phase (expansion or recession) produced over the period January 1979 to June 2011. Forecasts are constructed using "real-time" data as it appeared at the time the forecast would have been produced. The out-of-sample predictions are constructed from the posterior predictive density of two versions of the model in (2), which differ on the inclusion of state-level predictors. 